\title{
Unboundedness of thresholding and quantization for bandlimited signals
}

\author{
Holger Boche*, Ullrich J. Mönich \\ Technische Universität München, Lehrstuhl für Theoretische Informationstechnik, Arcisstraße 21, D-80290 München, Germany
}

\section{A R T I C L E I N F O}

\section{Article history:}

Received 13 September 2011

Received in revised form

2 January 2012

Accepted 5 April 2012

Available online 17 May 2012

\section{Keywords:}

Shannon sampling series

Threshold operator

Quantization

Paley-Wiener space

\begin{abstract}
A B S T R A C T
It is well-known that the Shannon sampling series is locally uniformly convergent for all signals in the Paley-Wiener space $\mathcal{P} \mathcal{W}_{\pi}^{1}$. An interesting question is how this good local approximation behavior is affected if the samples are disturbed by the non-linear threshold operator. This operator, which sets to zero all samples with absolute value smaller than some threshold, arises in the modeling of many applications, e.g., in wireless sensor networks. Moreover, it constitutes an essential part of a large class of quantizers, and consequently is important for all digital signal processing applications that involve conversion between analog and digital domains. In this paper, the approximation behavior of the Shannon sampling series that only uses the samples with absolute value larger than or equal to some threshold is analyzed. It is shown that there exists a signal in $\mathcal{P W}_{\pi}^{1}$ such that the local approximation error increases unboundedly as the threshold tends to zero. Moreover, for a fixed threshold, the local approximation error can grow arbitrarily large on the set of signals whose norm is bounded by one. With this, we generalize results of Butzer et al. that were given in the paper "On quantization, truncation and jitter errors in the sampling theorem and its generalizations," Signal Processing (2) 1980 [1]. We conclude the paper with a discussion about the differences in the reconstruction behavior between the sampling series which is truncated in the domain of the sampled signal, i.e., time-domain truncation, and the sampling series which is truncated in the range of the sampled signal. (c) 2012 Published by Elsevier B.V.
\end{abstract}

\section{Introduction}

A well known fact [2-4] about the convergence behavior of the Shannon sampling series for signals in $\mathcal{P} \mathcal{W}_{\pi}^{1}$ is expressed by the following theorem.

Theorem 1 (Brown's Theorem). For all $f \in \mathcal{P W}_{\pi}^{1}$ and $T>0$ fixed we have

$\lim _{N \rightarrow \infty}\left(\max _{t \in[-T, T]}\left|f(t)-\sum_{k=-N}^{N} f(k) \frac{\sin (\pi(t-k))}{\pi(t-k)}\right|\right)=0$.

\footnotetext{
* Corresponding author. Tel.: +498928923240

E-mail addresses: boche@tum.de (H. Boche), moenich@tum.de (U.J. Mönich).
}

This theorem plays a fundamental role in applications because it establishes the uniform convergence on compact subsets of $\mathbb{R}$ for a large class of signals, namely $\mathcal{P W} \mathcal{W}_{\pi}^{1}$, which is the largest space within the scale of PaleyWiener spaces. Unfortunately, it is not possible to extend the theorem in such a way that the uniform convergence holds on all of $\mathbb{R}$ for the space $\mathcal{P} \mathcal{W}_{\pi}^{1}$.

The reconstruction of bandlimited signals from their samples is important for many practical and theoretical applications. In digital signal processing, the Shannon sampling theorem is the theoretical foundation which creates the link between the continuous-time domain and the discrete-time domain. The Shannon sampling series has proven to be useful in other areas as well. In his "Lectures on Computation" [5], Richard Feynman discusses the theoretical foundations and concepts of 
classical and quantum computation and the Shannon sampling theorem is one important step in his argumentation.

The principle of digital signal processing relies on the fact that certain bandlimited signals can be perfectly reconstructed from their samples. However, this is only true if the sample values are known exactly. For various reasons this is not always the case in applications. For example, in digital signal processing applications the samples are not known exactly because the inevitable quantization process in analog to digital conversion has limited resolution only [6,7]. Due to its high practical importance, the analysis of the quantization error has gained a lot of attention in research [6]. A deterministic analysis of the quantization process is difficult because of the non-linear nature of the quantization operator. This fact was recognized for example in [8], where the authors write "Deceptively simple in its description and construction, the uniform quantizer has proved to be surprisingly difficult to analyze, precisely because of its inherent nonlinearity." This fact is also the reason why the quantization error is often treated probabilistically, and modeled as additive white noise $[9,10]$. However, it turned out that this noise model is not always satisfactory, because it can lead to false predictions [11,12]. In contrast, the deterministic analysis is difficult, but reveals some properties of the quantization process which cannot be analyzed with the additive noise description of the quantization error. Only a few papers conduct a deterministic analysis of the quantization process $[3,1,13]$.

In this paper, we provide for the space $\mathcal{P} \mathcal{W}_{\pi}^{1}$ the first rigorous deterministic analysis of the pointwise behavior of the Shannon sampling series, where the samples are disturbed by the threshold operator, which sets to zero all sample values with absolute value smaller than some threshold $\delta>0$. This operator constitutes an essential part of many quantization schemes, and thus the results obtained here are equally relevant for a large class of quantization operators. Of course there are also applications where the threshold operator is important on its own.

Wireless sensor networks are one possible application where the threshold operator is directly involved. In wireless sensor networks the sensors sample some bandlimited signal in space or time and then transmit the samples to the receiver $[14,15]$. Then, using these samples, the receiver tries to reconstruct the signal perfectly, or at least approximately if a perfect reconstruction is not possible. In order to save energy, it is common to let the sensors transmit only if the absolute value of the signal exceeds some threshold $\delta>0$. In this case, the receiver has to reconstruct the signal by using only the samples with absolute value larger than or equal to the threshold $\delta$.

By $A_{\delta}$ we denote the operator that maps the signal $f \in$ $\mathcal{P} \mathcal{W}_{\pi}^{1}$ to the approximation $A_{\delta} f$ of $f$, which is obtained by the Shannon sampling series that uses only the samples with an absolute value larger than or equal to the threshold $\delta$. A precise definition of $A_{\delta}$ will be given in Section 3.

In this paper we analyze the behavior of $A_{\delta} f$ in two ways. The first one is to analyze $A_{\delta} f$ for fixed threshold $\delta$ and vary $f \in \mathcal{P} \mathcal{W}_{\pi}^{1}$. In order to get meaningful results, we must additionally restrict the norm of the signals. We choose signals $f$ with norm $\|f\|_{\mathcal{P W}} \leq 1$. The second way is to analyze $A_{\delta} f$ for fixed $f \in \mathcal{P} \mathcal{W}_{\pi}^{1}$ as the threshold $\delta$ tends to zero. Intuitively one would expect that the approximation error is reduced if the threshold is decreased. However, as we will see in Section 4, this is not true generally. The threshold operator destroys the good local approximation behavior of the Shannon sampling series for $\mathcal{P} \mathcal{W}_{\pi}^{1}$. There are signals in $\mathcal{P} \mathcal{W}_{\pi}^{1}$ such that $\left(A_{\delta} f\right)(t)$ diverges for all $t \in \mathbb{R} \backslash \mathbb{Z}$ as $\delta \rightarrow 0$. Hence, for fixed $t \in \mathbb{R} \backslash \mathbb{Z}$, the approximation error $\left|f(t)-\left(A_{\delta} f\right)(t)\right|$ can grow arbitrarily large. This result improves a result which was recently obtained for the global behavior of $A_{\delta} f[16]$.

\section{Notation}

In order to continue, we need some notation. Let $\hat{f}$ denote the Fourier transform of a function $f$, where $\hat{f}$ is to be understood in the distributional sense. $L^{p}(\mathbb{R})$, $1 \leq p<\infty$, is the space of all to the $p$ th power Lebesgue integrable functions on $\mathbb{R}$, with the usual norm $\|\cdot\|_{p}$, and $L^{\infty}(\mathbb{R})$ the space of all functions for which the essential supremum norm $\|\cdot\|_{\infty}$ is finite. For $1 \leq p \leq \infty, \mathcal{P} \mathcal{W}_{\pi}^{p}$ denotes the Paley-Wiener space of signals $f$ with a representation $f(z)=1 /(2 \pi) \int_{-\pi}^{\pi} g(\omega) \mathrm{e}^{i z \omega} \mathrm{d} \omega, \quad z \in \mathbb{C}$, for some $g \in L^{p}[-\pi, \pi]$. If $f \in \mathcal{P} \mathcal{W}_{\pi}^{p}$ then $g(\omega)=\hat{f}(\omega)$. The norm for $\mathcal{P} \mathcal{W}_{\pi}^{p}, \quad 1 \leq p<\infty$, is given by $\|f\|_{\mathcal{P W}}=\left(1 /(2 \pi) \int_{-\pi}^{\pi}\right.$ $\left.|\hat{f}(\omega)|^{p} \mathrm{~d} \omega\right)^{1 / p}$. Moreover, we have $\|f\|_{\infty} \leq\|f\|_{\mathcal{P} \mathcal{W}_{\pi}^{1}}$, i.e., every signal in $\mathcal{P} \mathcal{W}_{\pi}^{1}$ is bounded on the real line.

\section{Motivation and contribution of the paper}

Before we state the main results, we introduce the threshold operator, discuss some of its basic properties, and substantiate the analyzed problem.

For complex numbers $z \in \mathbb{C}$, the threshold operator $\kappa_{\delta}$, $\delta>0$, is defined by

$\kappa_{\delta} z= \begin{cases}z, & |z| \geq \delta \\ 0, & |z|<\delta\end{cases}$

Furthermore, for continuous functions $f: \mathbb{R} \rightarrow \mathbb{C}$, we define the threshold operator $\Theta_{\delta}, \delta>0$, pointwise, i.e., $\left(\Theta_{\delta} f\right)(t)=\kappa_{\delta} f(t), t \in \mathbb{R}$.

In this paper, the threshold operator $\kappa_{\delta}$ is applied on the samples $\{f(k)\}_{k \in \mathbb{Z}}$ of signals $f \in \mathcal{P} \mathcal{W}_{\pi}^{1}$, which gives the disturbed samples $\left\{\kappa_{\delta} f(k)\right\}_{k \in \mathbb{Z}}$. This is, of course, equivalent to applying the threshold operator $\Theta_{\delta}$ on the signal $f$ itself and then taking the samples, i.e., $\left\{\left(\Theta_{\delta} f\right)(k)\right\}_{k \in \mathbb{Z}}$. Then, the resulting samples $\left\{\left(\Theta_{\delta} f\right)(k)\right\}_{k \in \mathbb{Z}}$ are used to build an approximation

$\left(A_{\delta} f\right)(t):=\sum_{\substack{k=-\infty \\|f(k)| \geq \delta}} f(k) \frac{\sin (\pi(t-k))}{\pi(t-k)}=\sum_{k=-\infty}^{\infty}\left(\Theta_{\delta} f\right)(k) \frac{\sin (\pi(t-k))}{\pi(t-k)}$

of the original signal $f$. By $A_{\delta}$ we denote the operator that maps $f \in \mathcal{P} \mathcal{W}_{\pi}^{1}$ to $A_{\delta} f$ according to (1). For $f \in \mathcal{P} \mathcal{W}_{\pi}^{1}$ we have $\lim _{|t| \rightarrow \infty} f(t)=0$ by the Riemann-Lebesgue lemma [17, p. 105], and it follows that the series in (1) has only finitely many summands, which implies that 
$A_{\delta} f \in \mathcal{P} \mathcal{W}_{\pi}^{2} \subset \mathcal{P} \mathcal{W}_{\pi}^{1}$. In general, $A_{\delta} f$ is only an approximation of $f$, and we want the signal $A_{\delta} f$ to be close to $f$ if $\delta$ is sufficiently small. The peak value of the approximation error $\left\|f-A_{\delta} f\right\|_{\infty}$ is one possible way to measure this closeness, another is the pointwise approximation error $\left|f(t)-\left(A_{\delta} f\right)(t)\right|, \quad t \in \mathbb{R}$. Since the series in (1) uses all "important" samples of the signal, i.e., all samples that are larger than or equal to $\delta$, one could expect $A_{\delta} f$ to have an approximation behavior similar to the Shannon sampling series. But, as we will see, $A_{\delta} f$ exhibits a significantly different behavior.

Some properties of $A_{\delta}$ which complicate the analysis of the threshold operator are as follows:

1. For each $\delta>0$ fixed, $A_{\delta}$ is a non-linear operator.

2. For each $\delta>0$ fixed, the operator $A_{\delta}:\left(\mathcal{P W} \mathcal{W}_{\pi}^{1},\|\cdot\|_{\mathcal{P W}}^{1}\right) \rightarrow$ $\left(\mathcal{P W} \mathcal{W}_{\pi}^{1},\|\cdot\|_{\infty}\right)$ is discontinuous, i.e., there exist a function $f \in \mathcal{P W}_{\pi}^{1}$ and a constant $C_{1}$ such that for every $\epsilon>0$ there exists a function $g \in \mathcal{P W}_{\pi}^{1}$ satisfying $\|f-g\|_{\mathcal{P W}}<\epsilon$ such that $\left\|A_{\delta} f-A_{\delta} g\right\|_{\infty} \geq C_{1}$. This implies that $A_{\delta}:\left(\mathcal{P} \mathcal{W}_{\pi}^{1},\|\cdot\|_{\mathcal{P W}}\right) \rightarrow\left(\mathcal{P} \mathcal{W}_{\pi}^{1},\|\cdot\|_{\mathcal{P W}}\right)$ is discontinuous.

3. For some $f \in \mathcal{P} \mathcal{W}_{\pi}^{1}$, the operator $A_{\delta}$ is also discontinuous with respect to $\delta$, i.e., there exist a function $f \in$ $\mathcal{P W}_{\pi}^{1}$ and a $t \in \mathbb{R}$ such that $\lim _{h \rightarrow 0}\left(A_{\delta+h} f\right)(t) \neq\left(A_{\delta} f\right)(t)$.

Remark 1. Note that a linear operator is continuous if and only if it is bounded. Since $A_{\delta}$ is a non-linear operator, continuity and boundedness are no longer equivalent. However, in Theorem 2 we will see that, for $0<\delta<1 / 3$, $A_{\delta}$ is also an unbounded operator.

The fact that $A_{\delta} f$ has only finitely many samples can be interpreted as a truncation of the Shannon sampling series

$\sum_{k=-\infty}^{\infty} f(k) \frac{\sin (\pi(t-k))}{\pi(t-k)}$.

This truncation is controlled in the range of the signal, because only the samples $f(k), k \in \mathbb{Z}$, with absolute value larger than or equal to some threshold $\delta>0$ are taken into account. As $\delta$ tends to zero, more and more samples are used for the approximation. Normally, the Shannon sampling series is truncated in the domain of the signal by considering only the samples $f(k), k=-N, \ldots, N$. For this kind of truncation we have, according to Brown's theorem, the uniform convergence of

$\left(S_{N} f\right)(t):=\sum_{k=-N}^{N} f(k) \frac{\sin (\pi(t-k))}{\pi(t-k)}$

on compact subsets of $\mathbb{R}$ for all $f \in \mathcal{P} \mathcal{W}_{\pi}^{1}$ as $N$ goes to infinity. It follows that $\sup _{N \in \mathbb{N}}\left|\left(S_{N} f\right)(t)\right|<\infty$ for all $t \in[-T, T]$, which in turn implies, using the BanachSteinhaus Theorem [18, p. 98], that there exists a constant $C_{2}$ such that

$\sup _{\|f\|_{\mathcal{P} w_{\pi}^{1}} \leq 1 N \in \mathbb{N}} \sup _{\mid}\left|\left(S_{N} f\right)(t)\right| \leq C_{2}$

for all $t \in[-T, T]$.

In contrast, the behavior of $A_{\delta} f$ is completely different. The following results about the global approximation behavior of $A_{\delta} f$, which are stated in Theorems 2 and 3, were recently obtained in [16].

Theorem 2. For all $0<\delta<1 / 3$ we have

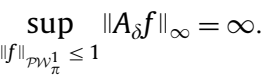

Theorem 2 shows that, for $0<\delta<1 / 3, A_{\delta}:\left(\mathcal{P} \mathcal{W}_{\pi}^{1},\|\cdot\|_{\mathcal{P W}}\right)$ $\rightarrow\left(\mathcal{P W} \mathcal{W}_{\pi}^{1},\|\cdot\|_{\infty}\right)$ is an unbounded operator. Thus, for any level $K>0$ we can find a signal $f \in \mathcal{P} \mathcal{W}_{\pi}^{1}$ with norm $\|f\|_{\mathcal{P W}} \leq 1$ such that $\left\|A_{\delta} f\right\|_{\infty}$ exceeds $K$. Furthermore, Theorem 2 implies that

$\sup _{\|f\|_{\mathcal{P} \mathcal{W}_{\pi}^{1}} \leq 1}\left\|f-A_{\delta} f\right\|_{\infty}=\infty$,

for every $0<\delta<1 / 3$, i.e., the peak approximation error can grow arbitrarily large.

Remark 2. Since the supremum in Theorem 2 is taken over signals with norm $\|f\|_{\mathcal{P} \mathcal{W}_{\pi}^{1}} \leq 1$, and $\|f\|_{\infty} \leq\|f\|_{\mathcal{P} \mathcal{W}_{\pi}^{1}}$, it is clear that the threshold $\delta$ must be less than or equal to one, because otherwise $A_{\delta} f \equiv 0$. The specific requirement in Theorem 2 that $0<\delta<1 / 3$ is due to technical reasons in the proof of the theorem.

Theorem 3. There exists a signal $f_{1} \in \mathcal{P} \mathcal{W}_{\pi}^{1}$ such that $\limsup _{\delta \rightarrow 0}\left\|A_{\delta} f_{1}\right\|_{\infty}=\infty$.

Theorem 3 shows that there exists a signal $f_{1} \in \mathcal{P} \mathcal{W}_{\pi}^{1}$ such that $\left\|A_{\delta} f_{1}\right\|_{\infty}$, i.e., the peak value of the approximation $A_{\delta} f_{1}$, increases unboundedly as the threshold $\delta$ tends to zero.

Both, Theorems 2 and 3, are concerned with the divergence of the supremum over $\mathbb{R}$ of the approximation $A_{\delta} f$. However, in certain applications a good local behavior of $A_{\delta} f$ on bounded intervals is sufficient and the global behavior is not relevant. This is the reason why we analyze the local behavior of $A_{\delta} f$ in this paper.

Remark 3. There is a seeming difference between the theorems in [16] and Theorems 2 and 3, because the results in [16] were stated for the case where the samples of the Shannon sampling series are disturbed by a quantization operator, performing a midtread quantization, and in this paper the threshold operator is used. However, the results are directly transferable because the key property of the quantization operator that was important for the proof in [16] is that all samples with absolute value less than the quantization threshold are set to zero, and this property equally holds for the threshold operator.

At the Strobl'11 conference, where we presented the results of this paper, we became aware that a closely related topic, which has recently been studied in the mathematical literature, is greedy approximation $[19,20]$. There, the approximation behavior of series like

$\sum_{|f(k)| \geq \delta} f(k) \mathrm{e}^{-i \omega k}, \quad \omega \in[-\pi, \pi]$,

where only the "important" Fourier coefficients are included, is analyzed, and the convergence of the series 
(4) is measured in the norm of the considered signal space. The results show that there exists a signal $f_{1} \in$ $\mathcal{P W}_{\pi}^{1}$ such that

$\limsup _{\delta \rightarrow 0} \int_{-\pi}^{\pi}\left|\sum_{|f(k)| \geq \delta} f_{1}(k) \mathrm{e}^{-i \omega k}\right| \mathrm{d} \omega=\infty$.

For practical applications the examination of the $L_{1}$-norm of (4), as is done in (5), is too restricting. For example, it can be shown that for every $0<\beta<1$ there exists a signal $f_{1} \in \mathcal{P} \mathcal{W}_{\beta \pi}^{1}$ for which, on the one hand, we have the divergence (5), but, on the other hand, we have the practically relevant uniform convergence

$\lim _{\delta \rightarrow 0} \max _{t \in \mathbb{R}}\left|f(t)-\sum_{|f(k)| \geq \delta} f_{1}(k) \phi(t-k)\right|=0$,

where $\phi$ is a suitable chosen reconstruction function. In this paper we analyze the case without oversampling.

Remark 4. Since

$\left(S_{N} f\right)(t)=\frac{1}{2 \pi} \int_{-\pi}^{\pi}\left(\sum_{k=-N}^{N} f(k) \mathrm{e}^{-i \omega k}\right) \mathrm{e}^{i \omega t} \mathrm{~d} \omega$,

where $S_{N}$ is defined as in (2), it follows that

$\left|\left(S_{N} f\right)(t)\right| \leq \frac{1}{2 \pi} \int_{-\pi}^{\pi}\left|\sum_{k=-N}^{N} f(k) \mathrm{e}^{-i \omega k}\right| \mathrm{d} \omega$.

That is, the divergence of the Shannon sampling series $\left(S_{N} f\right)(t)$ for some $t \in \mathbb{R}$ implies the divergence of the $L_{1}$ norm of

$\sum_{k=-N}^{N} f(k) \mathrm{e}^{-i \omega k}$

However, the converse is not true. There exist signals $f \in$ $\mathcal{P W}_{\pi}^{1}$ such that the $L_{1}$-norm of (6) diverges but-as Brown's theorem (Theorem 1) shows-the Shannon sampling series $\left(S_{N} f\right)(t)$ converges for all $t \in \mathbb{R}$. This shows that the bad behavior of (6) has no immediate consequence for the convergence behavior of the Shannon sampling series. In view of this, we cannot conclude the bad behavior of $\left(A_{\delta} f\right)(t)$ from the diverges of the $L_{1}$-norm of (4). However, we will see that there is a difference to the Shannon sampling series, because $\left(A_{\delta} f\right)(t)$ indeed diverges for some $f \in \mathcal{P} \mathcal{W}_{\pi}^{1}$ and all $t \in \mathbb{R} \backslash \mathbb{Z}$ as $\delta$ tends to zero.

\section{Unboundedness of the threshold operator}

We have seen that the threshold operator leads to a bad global reconstruction behavior of the Shannon sampling series. In this section we analyze whether this bad behavior is limited to the global behavior of the reconstruction or whether it is also locally present.

The next theorem is the analog theorem to Theorem 2 , and shows that the unboundedness of $A_{\delta} f$ on the set $\{f \in$ $\left.\mathcal{P} \mathcal{W}_{\pi}^{1}:\|f\|_{\mathcal{P W}}^{1} \leq 1\right\}$ is not only with respect to the supremum norm but also pointwise for every $t \in \mathbb{R} \backslash \mathbb{Z}$.
Theorem 4. For all $0<\delta<1 / 3$ and all $t \in \mathbb{R} \backslash \mathbb{Z}$ we have $\sup _{\|f\|_{P W_{\pi}^{1}} \leq 1}\left|\left(A_{\delta} f\right)(t)\right|=\infty$.

Although we have stated Theorem 4 for the threshold operator, the proof reveals that Theorem 4 is also true for all quantization operators that set all signal values below a certain threshold to zero, i.e., all quantization operators that behave like the threshold operator for small signal values. The uniform midtread quantization, for example, is a quantization operator that has this behavior.

The next theorem concerns the local behavior of $A_{\delta} f$ as the threshold $\delta$ tends to zero. It shows that $\left(A_{\delta} f\right)(t)$, $t \in \mathbb{R} \backslash \mathbb{Z}$, diverges as $\delta \rightarrow 0$ for some signal in $\mathcal{P} \mathcal{W}_{\pi}^{1}$.

Theorem 5. There exists a signal $f_{1} \in \mathcal{P} \mathcal{W}_{\pi}^{1}$ such that for all $t \in \mathbb{R} \backslash \mathbb{Z}$ we have

$\limsup _{\delta \rightarrow 0}\left|\left(A_{\delta} f_{1}\right)(t)\right|=\infty$.

Theorems 4 and 5 improve Theorems 2 and 3 respectively.

Remark 5. The divergence of $\left(A_{\delta} f\right)(t)$ between the integers is remarkable because the approximation behavior on the integer grid is best possible. For all $t \in \mathbb{Z}, f \in \mathcal{P} \mathcal{W}_{\pi}^{1}$, and $\delta>0$ we have $\left|f(t)-\left(A_{\delta} f\right)(t)\right|<\delta$.

It is not immediately clear how to prove Theorem 5 for the quantization operator that was discussed above, instead of the threshold operator. Nevertheless, we conjecture that the theorem is also true for the quantization operator.

We first prove Theorem 5 because the proof of Theorem 4 is simple, once we have the results from the first proof.

Proof of Theorem 5. The fact that the operator $A_{\delta}$ is discontinuous complicates the proof of Theorem 5 . In the proof we iteratively construct a sequence of $\mathcal{P} \mathcal{W}_{\pi}^{1}$-signals, which converges in the $\mathcal{P} \mathcal{W}_{\pi}^{1}$-norm to a signal $f_{1} \in \mathcal{P} \mathcal{W}_{\pi}^{1}$ that has the property (7).

For $0<\eta<1$ and $N \in \mathbb{N}$, consider the function

$f(t, \eta, N):=\sum_{k=-2 N+1}^{2 N-1} f(k, \eta, N) \frac{\sin (\pi(t-k))}{\pi(t-k)}$,

where

$f(k, \eta, N)= \begin{cases}(-1)^{k}\left(2(1-\eta)+\frac{1-\eta}{N} k\right), & -2 N<k<-N, \\ (-1)^{k}(1-\eta), & -N \leq k<0, \\ (-1)^{k}, & 0 \leq k \leq N, \\ (-1)^{k}\left(2-\frac{1}{N} k\right), & N<k<2 N .\end{cases}$

Additionally, for $0<\eta<1, N \in \mathbb{N}$, and $M \in \mathbb{N} \cup\{0\}, M<N$, the function

$g(t, \eta, N, M):=f(t, \eta, N)-\underbrace{\sum_{k=-M}^{-1}(1-\eta) \frac{(-1)^{k} \sin (\pi(t-k))}{\pi(t-k)}}_{=: u_{1}(t)}$ 


$$
-\underbrace{\sum_{k=0}^{M} \frac{(-1)^{k} \sin (\pi(t-k))}{\pi(t-k)}}_{=: u_{2}(t)}
$$

is needed. Note that, for $M=0$, the first sum in (9) is empty and thus $u_{1} \equiv 0$. We have

$\|g(\cdot, \eta, N, M)\|_{\mathcal{P W}}^{1} \leq\|f(\cdot, \eta, N)\|_{\mathcal{P W}}^{1}+\left\|u_{1}\right\|_{\mathcal{P W}}+\left\|u_{2}\right\|_{\mathcal{P W}}^{1}$.

The norm $\left\|u_{1}\right\|_{\mathcal{P} \mathcal{W}_{\pi}^{1}}$ is bounded above by

$\left\|u_{1}\right\|_{\mathcal{P} \mathcal{W}_{\pi}^{1}}<\frac{\pi}{2}+\log (M+1)$,

because $\left\|u_{1}\right\|_{\mathcal{P W}}=0$ for $M=0$, and

$$
\begin{aligned}
\left\|u_{1}\right\|_{\mathcal{P W}}{ }_{\pi}^{1} & =\frac{(1-\eta)}{2 \pi} \int_{-\pi}^{\pi}\left|\sum_{k=-M}^{-1} \mathrm{e}^{-i \omega k}(-1)^{k}\right| \mathrm{d} \omega \\
& =\frac{(1-\eta)}{2 \pi} \int_{-\pi}^{\pi}\left|\frac{1-\mathrm{e}^{i \omega M}}{1-\mathrm{e}^{i \omega}}\right| \mathrm{d} \omega \\
& <\frac{1}{\pi} \int_{0}^{\pi}\left|\frac{\sin \left(\frac{M}{2} \omega\right)}{\sin \left(\frac{\omega}{2}\right)}\right| \mathrm{d} \omega \\
& \leq \int_{0}^{\pi} \frac{\left|\sin \left(\frac{M}{2} \omega\right)\right|}{\omega} \mathrm{d} \omega \\
& =\int_{0}^{M} \frac{\left|\sin \left(\frac{\pi}{2} \omega\right)\right|}{\omega} \mathrm{d} \omega \\
& \leq \int_{0}^{1} \frac{\sin \left(\frac{\pi}{2} \omega\right)}{\omega} \mathrm{d} \omega+\int_{1}^{M} \frac{1}{\omega} \mathrm{d} \omega \\
& <\frac{\pi}{2}+\log (M)
\end{aligned}
$$

for $M>0$. A similar calculation shows that

$\left\|u_{2}\right\|_{\mathcal{P} \mathcal{W}_{\pi}^{1}}<\frac{\pi}{2}+\log (M+1)$.

In addition, we have

$\|f(\cdot, 0, N)\|_{\mathcal{P W}}<3$

and

$\lim _{\eta \rightarrow 0}\|f(\cdot, \eta, N)-f(\cdot, 0, N)\|_{\mathcal{P} \mathcal{W}_{\pi}^{1}}=0$.

Eq. (13) follows from the Appendix of [16], by observing that it is also possible to write a strict inequality in the last line of the last equation in [16]. Therefore, for every $N \in \mathbb{N}$, there exists an $\eta_{0}=\eta_{0}(N)>0$ such that

$\|f(\cdot, \eta, N)\|_{\mathcal{P W}_{\pi}^{1}}<3$ for all $\eta \leq \eta_{0}$.

By $\bar{\eta}(N)$ we denote the largest $\eta_{0}$ such that (14) is true. Combining (10)-(12) and (14), it follows that

$\|g(\cdot, \eta, N, M)\|_{\mathcal{P W}}<3+\pi+2 \log (M+1)$

for all $N \in \mathbb{N}, M \in \mathbb{N} \cup\{0\}, M<N$, and $\eta \leq \bar{\eta}(N)$.
Moreover, for $N \in \mathbb{N}, M \in \mathbb{N} \cup\{0\}, M<N, 0<\eta<1$, and all $\delta$ satisfying $\max (1-\eta, 1-1 / N)<\delta<1$, we have

$$
\begin{aligned}
\left(A_{\delta} g(\cdot, \eta, N, M)\right)\left(-\frac{1}{2}\right) & =\sum_{k=M+1}^{N} \frac{(-1)^{k} \sin \left(\pi\left(-\frac{1}{2}-k\right)\right)}{\pi\left(-\frac{1}{2}-k\right)} \\
& >\frac{1}{\pi} \sum_{k=M+1}^{N} \frac{1}{1+k} \\
& >\frac{1}{\pi} \sum_{k=M+1}^{N} \int_{k}^{k+1} \frac{1}{1+\tau} \mathrm{d} \tau \\
& =\frac{1}{\pi} \int_{M+1}^{N+1} \frac{1}{1+\tau} \mathrm{d} \tau \\
& =\frac{1}{\pi} \log \left(\frac{N+2}{M+2}\right) .
\end{aligned}
$$

The function

$h(t, \eta, N, M):=\frac{1}{3+\pi+2 \log (M+1)} g(t, \eta, N, M)$

will be a central building block of the desired function $f_{1}$. Due to (15), we have

$\|h(\cdot, \eta, N, M)\|_{\mathcal{P W}_{\pi}^{1}} \leq 1$

for all $\eta \leq \bar{\eta}(N)$.

Let $K>0$ and $M \in \mathbb{N} \cup\{0\}$ be arbitrary and choose $\underline{N}$ according to

$\underline{N}=\underline{N}(M, K)=(M+2) \mathrm{e}^{\pi K(3+\pi+2 \log (M+1))}-2$,

which implies that

$\frac{1}{\pi} \log \left(\frac{N+2}{M+2}\right)=K(3+\pi+2 \log (M+1))$.

Thus, for all $N \geq \underline{N}, 0<\eta<1$, and $\delta$ satisfying

$\max \left(\frac{1-\eta}{3+\pi+2 \log (M+1)}, \frac{1-\frac{1}{N}}{3+\pi+2 \log (M+1)}\right)<\delta<1$

it follows by (16) that

$\left(A_{\delta} h(\cdot, \eta, N, M)\right)\left(-\frac{1}{2}\right)>\frac{1}{3+\pi+2 \log (M+1)} \frac{1}{\pi} \log \left(\frac{N+2}{M+2}\right) \geq K$.

Now, we construct the function $f_{1}$ iteratively. Let $\epsilon_{1}=1$, $M_{1}=0$, and choose $N_{1}=\left\lceil\underline{N}\left(M_{1}, 2^{1+1} / \epsilon_{1}\right)\right\rceil$, where $\lceil t\rceil$ denotes the smallest integer that is larger than or equal to $t$. Then, for $\eta_{1}=\bar{\eta}\left(N_{1}\right)$ and whenever $\delta_{1}$ is chosen such that

$\max \left(\frac{1-\eta_{1}}{3+\pi}, \frac{1-\frac{1}{N_{1}}}{3+\pi}\right)<\delta_{1}<\frac{1}{3+\pi}$,

we have, using the abbreviation $\phi_{1}(t)=h\left(t, \eta_{1}, N_{1}, M_{1}\right)$, that $\left\|\phi_{1}\right\|_{\mathcal{P} \mathcal{W}_{\pi}^{1}} \leq 1$,

which follows from (17), and

$\left(A_{\delta_{1}} \phi_{1}\right)\left(-\frac{1}{2}\right)>\frac{2^{1+1}}{\epsilon_{1}}=2^{1+1}$.

Since only finitely many samples of $\phi_{1}$ are different from zero, it follows that $\phi_{1} \in \mathcal{P} \mathcal{W}_{\pi}^{2}$. In [21, Theorem 5] it 
was shown that $\lim _{\delta \rightarrow 0}\left\|A_{\delta} f-f\right\|_{\infty}=0$ for all $f \in \mathcal{P} \mathcal{W}_{\pi}^{2}$. Therefore, there exists a $\bar{\delta}_{2}>0$ such that

$$
\left|\left(A_{\delta} \phi_{1}\right)\left(-\frac{1}{2}\right)-\phi_{1}\left(-\frac{1}{2}\right)\right|<1
$$

for all $\delta \leq \bar{\delta}_{2}$. Next, let $0<\epsilon_{2}<\min \left(\epsilon_{1} / 2, \bar{\delta}_{2}\right), M_{2}=2 N_{1}$, $N_{2}=\left\lceil\underline{N}\left(M_{2}, 2^{2+1} / \epsilon_{2}\right)\right\rceil, \eta_{2}=\bar{\eta}\left(N_{2}\right)$, and define the function $\phi_{2}(t)=\phi_{1}(t)+\epsilon_{2} h\left(t, \eta_{2}, N_{2}, M_{2}\right)$. Then we have, for all $\delta_{2}$ with

$$
\begin{aligned}
& \max \left(\frac{\left(1-\eta_{2}\right) \epsilon_{2}}{3+\pi+2 \log \left(M_{2}+1\right)}, \frac{\left(1-\frac{1}{N_{2}}\right) \epsilon_{2}}{3+\pi+2 \log \left(M_{2}+1\right)}\right)<\delta_{2} \\
& \quad<\frac{\epsilon_{2}}{3+\pi+2 \log \left(M_{2}+1\right)},
\end{aligned}
$$

that

$$
\begin{aligned}
\left(A_{\delta_{2}} \phi_{2}\right)\left(-\frac{1}{2}\right)= & \sum_{k=-2 N_{1}+1}^{2 N_{1}-1}\left(\Theta_{\delta_{2}} \phi_{2}\right)(k) \frac{\sin \left(\pi\left(-\frac{1}{2}-k\right)\right)}{\pi\left(-\frac{1}{2}-k\right)} \\
& +\sum_{k=M_{2}+1}^{N_{2}}\left(\Theta_{\delta_{2}} \phi_{2}\right)(k) \frac{\sin \left(\pi\left(-\frac{1}{2}-k\right)\right)}{\pi\left(-\frac{1}{2}-k\right)} \\
= & \left(A_{\delta_{2}} \phi_{1}\right)\left(-\frac{1}{2}\right)+\frac{\epsilon_{2}}{3+\pi+2 \log \left(M_{2}+1\right)} \\
& \times \sum_{k=M_{2}+1}^{N_{2}} \frac{(-1)^{k} \sin \left(\pi\left(-\frac{1}{2}-k\right)\right)}{\pi\left(-\frac{1}{2}-k\right)} .
\end{aligned}
$$

Since $\delta_{2}<\bar{\delta}_{2}$, it follows by (19) that

$$
\left|\left(A_{\delta_{2}} \phi_{1}\right)\left(-\frac{1}{2}\right)-\phi_{1}\left(-\frac{1}{2}\right)\right|<1
$$

and consequently

$$
\begin{aligned}
& \left(A_{\delta_{2}} \phi_{2}\right)\left(-\frac{1}{2}\right)>\frac{\epsilon_{2}}{3+\pi+2 \log \left(M_{2}+1\right)} \\
& \quad \times \sum_{k=M_{2}+1}^{N_{2}} \frac{(-1)^{k} \sin \left(\pi\left(-\frac{1}{2}-k\right)\right)}{\pi\left(-\frac{1}{2}-k\right)}-\left|\phi_{1}\left(-\frac{1}{2}\right)\right|-1 \\
& \quad \geq \frac{\epsilon_{2}}{3+\pi+2 \log \left(M_{2}+1\right)}\left(3+\pi+2 \log \left(M_{2}+1\right)\right) \frac{8}{\epsilon_{2}}-2 \\
& \quad=2^{2+1}-2,
\end{aligned}
$$

where we used (17), which implies that $\left\|\phi_{1}\right\|_{\infty} \leq$ $\left\|\phi_{1}\right\|_{\mathcal{P} \mathcal{W}_{\pi}^{1}} \leq 1$.

Again, there exists a $\bar{\delta}_{3}>0$ such that

$$
\left|\left(A_{\delta} \phi_{2}\right)\left(-\frac{1}{2}\right)-\phi_{2}\left(-\frac{1}{2}\right)\right| \leq 1
$$

for all $\delta<\bar{\delta}_{3}$. Next, let $0<\epsilon_{3}<\min \left(\epsilon_{2} / 2, \bar{\delta}_{3}\right), M_{3}=2 N_{2}$, $N_{3}=\left\lceil\underline{N}\left(M_{3}, 2^{3+1} / \epsilon_{3}\right)\right\rceil, \quad \eta_{3}=\bar{\eta}\left(N_{3}\right), \quad$ and define $\phi_{3}(t)=$ $\phi_{2}(t)+\epsilon_{3} h\left(t, \eta_{3}, N_{3}, M_{3}\right)$. Then we have, for all $\delta_{3}$ with

$$
\begin{aligned}
& \max \left(\frac{\left(1-\eta_{3}\right) \epsilon_{3}}{3+\pi+2 \log \left(M_{3}+1\right)}, \frac{\left(1-\frac{1}{N_{3}}\right) \epsilon_{3}}{3+\pi+2 \log \left(M_{3}+1\right)}\right)<\delta_{3} \\
& \quad<\frac{\epsilon_{3}}{3+\pi+2 \log \left(M_{3}+1\right)},
\end{aligned}
$$

that

$$
\begin{aligned}
& \left(A_{\delta_{3}} \phi_{3}\right)\left(-\frac{1}{2}\right)=\sum_{k=-2 N_{2}+1}^{2 N_{2}-1}\left(\Theta_{\delta_{3}} \phi_{3}\right)(k) \frac{\sin \left(\pi\left(-\frac{1}{2}-k\right)\right)}{\pi\left(-\frac{1}{2}-k\right)} \\
& +\sum_{k=M_{3}+1}^{N_{3}}\left(\Theta_{\delta_{3}} \phi_{3}\right)(k) \frac{\sin \left(\pi\left(-\frac{1}{2}-k\right)\right)}{\pi\left(-\frac{1}{2}-k\right)}
\end{aligned}
$$

$$
\begin{gathered}
=\left(A_{\delta_{3}} \phi_{2}\right)\left(-\frac{1}{2}\right)+\frac{\epsilon_{3}}{3+\pi+2 \log \left(M_{3}+1\right)} \\
\times \sum_{k=M_{3}+1}^{N_{3}} \frac{(-1)^{k} \sin \left(\pi\left(-\frac{1}{2}-k\right)\right)}{\pi\left(-\frac{1}{2}-k\right)} .
\end{gathered}
$$

Since $\delta_{3}<\bar{\delta}_{3}$ it follows by (20) that

$$
\left|\left(A_{\delta_{3}} \phi_{2}\right)\left(-\frac{1}{2}\right)-\phi_{2}\left(-\frac{1}{2}\right)\right|<1
$$

and consequently

$$
\begin{aligned}
\left(A_{\delta_{3}} \phi_{3}\right) & \left(-\frac{1}{2}\right) \geq \frac{\epsilon_{3}}{3+\pi+2 \log \left(M_{3}+1\right)} \\
& \times \sum_{k=M_{3}+1}^{N_{3}} \frac{(-1)^{k} \sin \left(\pi\left(-\frac{1}{2}-k\right)\right)}{\pi\left(-\frac{1}{2}-k\right)}-\left|\phi_{2}\left(-\frac{1}{2}\right)\right|-1 .
\end{aligned}
$$

Furthermore, using (18), we have that

$\left\|\phi_{2}\right\|_{\mathcal{P W}}^{1} \leq\left\|\phi_{1}\right\|_{\mathcal{P W}}^{1}+\epsilon_{2}\left\|h\left(\cdot, \eta_{2}, N_{2}, M_{2}\right)\right\|_{\mathcal{P W}}<1+\frac{1}{2}$

and

$$
\begin{aligned}
& \left\|\phi_{3}\right\|_{\mathcal{P W} \mathcal{W}_{\pi}^{1}} \leq\left\|\phi_{2}\right\|_{\mathcal{P W}}+\epsilon_{3}\left\|h\left(\cdot, \eta_{3}, N_{3}, M_{3}\right)\right\|_{\mathcal{P W}}<1+\frac{1}{2}+\frac{1}{4} \\
& <\sum_{k=0}^{\infty} \frac{1}{2^{k}}=2
\end{aligned}
$$

which leads to

$\left(A_{\delta_{3}} \phi_{3}\right)\left(-\frac{1}{2}\right)>2^{3+1}-3$.

Now, using the same procedure again and again, we can iteratively construct the sequence of functions $\phi_{4}, \phi_{5}, \ldots$, and, by induction, we find that

$\phi_{k}(t)=\sum_{l=1}^{k} \epsilon_{l} h\left(t, \eta_{l}, N_{l}, M_{l}\right), \quad k \in \mathbb{N}$

Since our choice of $\epsilon_{l}, l \in N$, ensures that $\left\{\phi_{k}\right\}_{k \in \mathbb{N}}$ is a Cauchy sequence in $\mathcal{P} \mathcal{W}_{\pi}^{1}$ it follows that there is a function $f_{1} \in \mathcal{P} \mathcal{W}_{\pi}^{1}$ with

$\lim _{k \rightarrow \infty}\left\|f_{1}-\phi_{k}\right\|_{\mathcal{P W}}=0$

and $\left\|f_{1}\right\|_{\mathcal{P} W_{\tau}^{1}} \leq 2$. Note that $\left(A_{\delta_{r}} f_{1}\right)(t)=\left(A_{\delta_{r}} \phi_{r}\right)(t)$ by the special construction of $\phi_{r}$. Using induction again leads to $\left(A_{\delta_{r}} f_{1}\right)\left(-\frac{1}{2}\right)>2^{r+1}-3, \quad r \in \mathbb{N}$,

which implies that

$\limsup _{\delta \rightarrow 0}\left(A_{\delta} f_{1}\right)\left(-\frac{1}{2}\right)=\infty$.

In the last step of the proof we show that (21) implies the assertion of the theorem. Let $t_{1}, t_{2} \in \mathbb{R} \backslash \mathbb{Z}$ be arbitrary. Then we have

$$
\begin{aligned}
& \left|\frac{1}{\sin \left(\pi t_{1}\right)}\left(A_{\delta} f_{1}\right)\left(t_{1}\right)-\frac{1}{\sin \left(\pi t_{2}\right)}\left(A_{\delta} f_{1}\right)\left(t_{2}\right)\right| \\
& \quad=\left|\sum_{k=-\infty}^{\infty}\left(\Theta_{\delta} f_{1}\right)(k) \frac{(-1)^{k}}{\pi\left(t_{1}-k\right)}-\sum_{k=-\infty}^{\infty}\left(\Theta_{\delta} f_{1}\right)(k) \frac{(-1)^{k}}{\pi\left(t_{2}-k\right)}\right| \\
& \quad=\left|\sum_{k=-\infty}^{\infty}\left(\Theta_{\delta} f_{1}\right)(k) \frac{(-1)^{k}\left(t_{2}-t_{1}\right)}{\pi\left(t_{1}-k\right)\left(t_{2}-k\right)}\right| \\
& \quad \leq \sum_{k=-\infty}^{\infty}\left|\left(\Theta_{\delta} f_{1}\right)(k)\right| \frac{\left|t_{2}-t_{1}\right|}{\pi\left|t_{1}-k\right|\left|t_{2}-k\right|}
\end{aligned}
$$




$$
\begin{aligned}
& \leq\left\|f_{1}\right\|_{\mathcal{P W}} \frac{\left|t_{2}-t_{1}\right|}{\pi} \sum_{k=-\infty}^{\infty} \frac{1}{\left|t_{1}-k\right|\left|t_{2}-k\right|} \\
& =\left\|f_{1}\right\|_{\mathcal{P W} \mathcal{W}_{\pi}^{1}} \frac{\left|t_{2}-t_{1}\right|}{\pi} C_{3}\left(t_{1}, t_{2}\right),
\end{aligned}
$$

where $C_{3}\left(t_{1}, t_{2}\right)<\infty$ is a constant that depends only on $t_{1}$ and $t_{2}$. Choosing $t_{1}=-1 / 2$ and $t=t_{2} \in \mathbb{R} \backslash \mathbb{Z}$ arbitrary and using (21), we obtain

$\limsup _{\delta \rightarrow 0}\left|\left(A_{\delta} f_{1}\right)(t)\right|=\infty$,

which completes the proof.

Proof of Theorem 4. Let $0<\delta<1 / 3$ be arbitrary but fixed. Moreover, for $N \in \mathbb{N}, N \geq 2$, we know from the proof of Theorem 5 that there exits an $\eta_{N}>0$ such that $\left\|f\left(\cdot, \eta_{N}, N\right)\right\|_{\mathcal{P W} \mathcal{W}^{1}}<3$, where $f$ is the function that was defined in (8). Next, choose some $\epsilon_{N}$ that satisfies $\epsilon_{N}>\delta$, $\epsilon_{N} \leq 1 / 3$, and $\max \left\{\epsilon_{N}\left(1-\eta_{N}\right), \epsilon_{N}(1-1 / N)\right\}<\delta$. Then the norm of

$u_{N}(t):=\epsilon_{N} f\left(t, \eta_{N}, N\right)$

satisfies

$\left\|u_{N}\right\|_{\mathcal{P W}}^{1}=\left|\epsilon_{N}\right|\left\|f\left(\cdot, \eta_{N}, N\right)\right\|_{\mathcal{P W}}^{1} \leq 1$.

Furthermore, we have

$$
\begin{aligned}
& \left(A_{\delta} u_{N}\right)\left(-\frac{1}{2}\right)=\epsilon_{N} \sum_{k=0}^{N}(-1)^{k} \frac{\sin \left(\pi\left(-\frac{1}{2}-k\right)\right)}{\pi\left(-\frac{1}{2}-k\right)} \\
& \geq \frac{\epsilon_{N}}{\pi} \sum_{k=0}^{N} \frac{1}{k} \\
& >\frac{\delta}{\pi} \log (N+2) .
\end{aligned}
$$

Since $N \in \mathbb{N}, N \geq 2$, was arbitrary, it follows that

$$
\sup _{\|f\|_{\mathcal{P W}}^{1} \leq 1}\left(A_{\delta} f\right)\left(-\frac{1}{2}\right)=\infty
$$

for all $0<\delta<1 / 3$. The assertion for arbitrary $t \in \mathbb{R} \backslash \mathbb{Z}$ can be obtained by using the same arguments that were used at the end of the proof of Theorem 5 .

\section{Discussion}

Truncation is a very important operator, not because it is an integral part in the quantization process. In Section 3 we have briefly given the interpretation of

$$
\left(A_{\delta} f\right)(t)=\sum_{\substack{k=-\infty \\|f(k)| \geq \delta}}^{\infty} f(k) \frac{\sin (\pi(t-k))}{\pi(t-k)},
$$

as a truncation of the Shannon sampling series

$$
\sum_{k=-\infty}^{\infty} f(k) \frac{\sin (\pi(t-k))}{\pi(t-k)},
$$

which is controlled in the range of the signal, because only the samples $f(k), k \in \mathbb{Z}$, with absolute value larger than or equal to the threshold $\delta>0$ are taken into account. This is in contrast to the usual truncation of the Shannon sampling series which is done in the domain of the signal, by considering only the samples $f(k)$, $k=-N, \ldots, N$. This kind of truncation leads to the finite sampling series

$\left(S_{N} f\right)(t)=\sum_{k=-N}^{N} f(k) \frac{\sin (\pi(t-k))}{\pi(t-k)}$,

which is relevant for practical applications, where only a finite number of samples can be considered in the reconstruction.

In the following discussion we will compare the reconstruction behavior of the Shannon sampling series for both types of truncation and point out the differences. First, we contrast the global behavior of $S_{N} f$ and $A_{\delta} f$. For the truncation in the domain of the signal, we have the well-known result [22] that

$\left\|S_{N} f\right\|_{\infty} \leq C_{4} \log (N)\|f\|_{\mathcal{P W}}$,

i.e., for fixed $N$, the peak value of $S_{N} f$ is bounded above, and it follows that

$$
\sup _{\|f\|_{\mathcal{P W} 1}^{1} \leq 1}\left\|S_{N} f\right\|_{\infty} \leq C_{4} \log (N) .
$$

For the truncation controlled in the range we do not have such a behavior. As was shown in [16], for all $0<\delta<1 / 3$, we have

$$
\sup _{\| \mathcal{P} w_{\pi}^{1} \leq 1}\left\|A_{\delta} f\right\|_{\infty}=\infty
$$

Hence, for fixed threshold $\delta, 0<\delta<1 / 3$, the peak value of $A_{\delta} f$ can grow arbitrarily large, i.e., for every $C_{5}>0$ there exists a signal $f_{1} \in \mathcal{P} \mathcal{W}_{\pi}^{1}$ with $\left\|f_{1}\right\|_{\mathcal{P W}_{\pi}^{1}} \leq 1$, such that $\left\|A_{\delta} f_{1}\right\|_{\infty}>C_{5}$.

Next, we discuss the local reconstruction behavior. For the truncation in the domain of the signal, we have Brown's Theorem (Theorem 1) which states the local uniform convergence of $S_{N} f$ for all signals $f \in \mathcal{P} \mathcal{W}_{\pi}^{1}$, as more and more samples of the signal are used in the reconstruction, i.e., as $N$ goes to infinity. In contrast, Theorem 5 shows that the reconstruction process $A_{\delta} f$, which is controlled by a truncation in the range of the sampled signal, does not possess this good reconstruction behavior. For fixed $t \in \mathbb{R} \backslash \mathbb{Z}$, we have lim sup $\operatorname{su}_{\delta \rightarrow 0} \mid$ $\left(A_{\delta} f_{1}\right)(t) \mid=\infty$ for some signal $f_{1} \in \mathcal{P} \mathcal{W}_{\pi}^{1}$. Thus, $\left|\left(A_{\delta} f_{1}\right)(t)\right|$ grows arbitrarily large as more and more samples of the signal are used in the reconstruction, i.e., as the threshold $\delta$ is reduced to zero.

Remark 6. For the truncation in the domain of the sampled signal, the peak value of the difference of the truncated sampling series for two signals can be controlled, in the sense that for all $\epsilon>0$ and all $f_{1}, f_{2} \in \mathcal{P} \mathcal{W}_{\pi}^{1}$, we have $\left\|S_{N} f_{1}-S_{N} f_{2}\right\|_{\infty} \leq C_{4} \log (N) \epsilon$ if $\left\|f_{1}-f_{2}\right\|_{\mathcal{P W}}<\epsilon$. This follows directly from (23). For the truncation that is controlled in the range of the sampled signal, the same result cannot hold, as the following counterexample shows. Choose $\epsilon=1, f_{1} \equiv 0$, and $0<\delta<1 / 3$. Then, according to (24), for every $C_{6}>0$, we can find a signal $f_{2} \in \mathcal{P} \mathcal{W}_{\pi}^{1}$ with $\left\|f_{2}\right\|_{\mathcal{P} \mathcal{W}_{\pi}^{1}} \leq 1$ such that $\left\|A_{\delta} f_{1}-A_{\delta} f_{2}\right\|_{\infty}=\left\|A_{\delta} f_{2}\right\|_{\infty}>C_{6}$, although $\left\|f_{1}^{\pi}-f_{2}\right\|_{\mathcal{P W}}^{1}=\left\|f_{2}\right\|_{\mathcal{P W}} \leq 1$.

Next, we will discuss the asymptotic speed of divergence. From (23) it follows, using some additional arguments, involving the Banach-Steinhaus Theorem and 
density arguments, that

$\lim _{N \rightarrow \infty} \frac{\left\|S_{N} f\right\|_{\infty}}{\log (N)}=0$

for all $f \in \mathcal{P} \mathcal{W}_{\pi}^{1}$, which shows that the peak value of $S_{N} f$ does asymptotically grow slower than $\log (N)$. It is natural to ask whether a similar result is also true for the truncation that is controlled in the range of the sampled signal.

Question 1. Does there exist a monotonically decreasing function $\phi_{1}$ with $\lim _{\delta \rightarrow 0} \phi_{1}(\delta)=\infty$ such that

$\limsup _{\delta \rightarrow 0} \frac{\left\|A_{\delta} f\right\|_{\infty}}{\phi_{1}(\delta)}=0$

for all $f \in \mathcal{P W}_{\pi}^{1}$ ?

The answer to this question is open.

From a practical point of view, the mere signal reconstruction is often not enough and the interest is rather in the output of a stable linear time-invariant (LTI) system. In [21] the approximation of stable LTI systems by sampling series with samples that are disturbed by the threshold operator was analyzed. It was shown that if $T$ : $\mathcal{P W}_{\pi}^{1} \rightarrow \mathcal{P} \mathcal{W}_{\pi}^{1}$ is a stable LTI system, $0<\delta<1 / 3$, and $t \in \mathbb{R}$, then we have

$$
\sup _{\|f\|_{\mathcal{P} W_{\pi}^{1}} \leq 1}\left|\left(T A_{\delta} f\right)(t)\right|<\infty
$$

if and only if

$$
\sum_{k=-\infty}^{\infty}\left|h_{T}(t-k)\right|<\infty,
$$

where $h_{T}=T$ sinc is the impulse response of the stable LTI system $T$. For a precise definition of a stable LTI system, see for example [21]. From this result, the following question arises.

Question 2. If (25) is not fulfilled, does there exist a signal $f_{1} \in \mathcal{P} \mathcal{W}_{\pi}^{1}$ such that $\lim \sup _{\delta \rightarrow 0}\left|\left(T A_{\delta} f_{1}\right)(t)\right|=\infty$ ?

Theorem 5, which gives a positive answer to this question for the special case where the system $T$ is the ideal low-pass filter, may be an indication that this question can be answered in the affirmative for general stable LTI systems.

The analysis of thresholding and quantization is difficult because of the non-linear nature of both operations. This is why stochastic approaches are often used to linearize the problem. The proofs in this paper show that the findings could not have been derived with a stochastic model. In the proof of Theorem 5, we furthermore gave an explicit procedure for the construction of a divergence creating signal $f_{1}$. This leads to the following question.

Question 3. Is it true that, in a topological sense, almost all signals in $\mathcal{P W}_{\pi}^{1}$ have the same problematic behavior?

In this paper we treated the problems for signals in $\mathcal{P} \mathcal{W}_{\pi}^{1}$, i.e., deterministic signals. For certain applications it is desirable to have results for stochastic processes also. In [23], the mean-square convergence behavior of the Shannon sampling series was analyzed for bandlimited continuous-time wide-sense stationary stochastic processes.
It would be interesting to study the approximation of such stochastic processes if the samples are additionally disturbed by the threshold operator. In this analysis the threshold operator would still be treated deterministically.

Finally, we come to the question if we can further strengthen the divergence statements. For the global behavior of the Shannon sampling series without thresholding we have the following result. There exists a signal $f_{1} \in \mathcal{P} \mathcal{W}_{\pi}^{1}$ such that $\lim \sup _{N \rightarrow \infty}\left\|S_{N} f_{1}\right\|_{\infty}=\infty$ [22]. In this statement we have a "lim sup", just like in Theorems 3 and 5 .

Question 4. Do the results still hold if the "lim sup" is replaced by "lim"?

For stochastic processes, a divergence result was given in [23], where a "lim" is used.

\section{Acknowledgments}

We would like to thank the anonymous reviewers for their interesting and helpful comments, which led to many of the open questions above.

\section{References}

[1] P.L. Butzer, W. Splettstößer, On quantization, truncation and jitter errors in the sampling theorem and its generalizations, Signal Processing 2 (2) (1980) 101-112.

[2] J.L. Brown Jr, On the error in reconstructing a non-bandlimited function by means of the bandpass sampling theorem, Journal of Mathematical Analysis and Applications 18 (1967) 75-84. (Erratum, Journal of Mathematical Analysis and Applications 21 (1968) 699).

[3] P.L. Butzer, W. Splettstößer, R.L. Stens, The sampling theorem and linear prediction in signal analysis, Jahresbericht der Deutschen Mathematiker-Vereinigung 90 (1) (1988) 1-70.

[4] P.L. Butzer, R.L. Stens, Sampling theory for not necessarily bandlimited functions: a historical overview, SIAM Review 34 (1) (1992) 40-53.

[5] R.P. Feynman, Feynman Lectures on Computation, Penguin Books, 1999.

[6] R.M. Gray, D.L. Neuhoff, Quantization, IEEE Transactions on Information Theory 44 (6) (1998) 2325-2383.

[7] I. Daubechies, R.A. DeVore, C.S. Güntürk, V.A. Vaishampayan, A/D conversion with imperfect quantizers, IEEE Transactions on Information Theory 52 (3) (2006) 874-885.

[8] R.M. Gray, T.G. Stockham, Dithered quantizers, IEEE Transactions on Information Theory 39 (3) (1993) 805-812.

[9] W.R. Bennett, Spectra of quantized signals, Bell System Technical Journal 27 (1948) 446-472.

[10] A. Papoulis, Error analysis in sampling theory, Proceedings of the IEEE 54 (7) (1966) 947-955.

[11] Z. Cvetković, M. Vetterli, Error-rate characteristics of oversampled analog-to-digital conversion, IEEE Transactions on Information Theory 44 (5) (1998) 1961-1964.

[12] N. Thao, M. Vetterli, Deterministic analysis of oversampled A/D conversion and decoding improvement based on consistent estimates, IEEE Transactions on Signal Processing 42 (3) (1994) 519-531.

[13] P.L. Butzer, A survey of the Whittaker-Shannon sampling theorem, Journal of Mathematical Research and Exposition 3 (1983) 185-212.

[14] P. Ishwar, A. Kumar, K. Ramchandran, Distributed sampling for dense sensor networks: a "bit-conservation principle", in: Information Processing in Sensor Networks: Second International Workshop, IPSN 2003, Lecture Notes in Computer Science, Springer Verlag, 2003, pp. 17-31

[15] A. Kumar, P. Ishwar, K. Ramchandran, On distributed sampling of smooth non-bandlimited fields, in: IPSN '04: Proceedings of the 
Third International Symposium on Information Processing in Sensor Networks, ACM Press, 2004, pp. 89-98.

[16] H. Boche, U.J. Mönich, Behavior of the quantization operator for bandlimited, nonoversampled signals, IEEE Transactions on Information Theory 56 (5) (2010) 2433-2440.

[17] L. Grafakos, Classical Fourier Analysis, 2nd edition, Springer, 2008.

[18] W. Rudin, Real and Complex Analysis, 3rd edition, McGraw-Hill, 1987.

[19] V.N. Temlyakov, Greedy expansions in Banach spaces, Advances in Computational Mathematics 26 (4) (2007) 431-449.

[20] P. Wojtaszczyk, Greedy algorithm for general biorthogonal systems, Journal of Approximation Theory 107 (2) (2000) 293-314.
[21] H. Boche, U.J. Mönich, Complete characterization of stable bandlimited systems under quantization and thresholding, IEEE Transactions on Signal Processing 57 (12) (2009) 4699-4710.

[22] H. Boche, U.J. Mönich, There exists no globally uniformly convergent reconstruction for the Paley-Wiener space $\mathcal{P} \mathcal{W}_{\pi}^{1}$ of bandlimited functions sampled at Nyquist rate, IEEE Transactions on Signal Processing 56 (7) (2008) 3170-3179.

[23] H. Boche, U.J. Mönich, Approximation of wide-sense stationary stochastic processes by Shannon sampling series, IEEE Transactions on Information Theory 56 (12) (2010) 6459-6469. 